\title{
Seeing in the dark
}

Citation for published version (APA):

de Graaf, T. A., Duecker, F., Stankevich, Y., Ten Oever, S., \& Sack, A. T. (2017). Seeing in the dark: Phosphene thresholds with eyes open versus closed in the absence of visual inputs. Brain stimulation, 10(4), 828-835. https://doi.org/10.1016/j.brs.2017.04.127

Document status and date:

Published: 01/07/2017

DOI:

10.1016/j.brs.2017.04.127

Document Version:

Publisher's PDF, also known as Version of record

\section{Document license:}

Taverne

\section{Please check the document version of this publication:}

- A submitted manuscript is the version of the article upon submission and before peer-review. There can be important differences between the submitted version and the official published version of record.

People interested in the research are advised to contact the author for the final version of the publication, or visit the DOI to the publisher's website.

- The final author version and the galley proof are versions of the publication after peer review.

- The final published version features the final layout of the paper including the volume, issue and page numbers.

Link to publication

\footnotetext{
General rights rights.

- You may freely distribute the URL identifying the publication in the public portal. please follow below link for the End User Agreement:

www.umlib.nl/taverne-license

Take down policy

If you believe that this document breaches copyright please contact us at:

repository@maastrichtuniversity.nl

providing details and we will investigate your claim.
}

Copyright and moral rights for the publications made accessible in the public portal are retained by the authors and/or other copyright owners and it is a condition of accessing publications that users recognise and abide by the legal requirements associated with these

- Users may download and print one copy of any publication from the public portal for the purpose of private study or research.

- You may not further distribute the material or use it for any profit-making activity or commercial gain

If the publication is distributed under the terms of Article $25 \mathrm{fa}$ of the Dutch Copyright Act, indicated by the "Taverne" license above, 


\title{
Seeing in the dark: Phosphene thresholds with eyes open versus closed in the absence of visual inputs
}

\author{
T.A. de Graaf a, b, *,1, F. Duecker ${ }^{\text {a, b, } 1}$, Y. Stankevich ${ }^{\text {c }}$, S. ten Oever ${ }^{\text {a, b }}$, A.T. Sack ${ }^{\text {a, b }}$ \\ a Department of Cognitive Neuroscience, Faculty of Psychology and Neuroscience, Maastricht University, Maastricht, The Netherlands \\ ${ }^{\mathrm{b}}$ Maastricht Brain Imaging Centre, Maastricht, The Netherlands \\ ${ }^{\mathrm{c}}$ Department of Psychology, Institute of Clinical Psychology and Psychotherapy, Technische Universität Dresden, Dresden, Germany
}

\section{A R T I C L E I N F O}

\section{Article history:}

Received 17 November 2016

Received in revised form

24 February 2017

Accepted 23 April 2017

Available online 3 May 2017

\section{Keywords:}

Phosphene

Threshold

Alpha

Oscillations

Excitability

Transcranial magnetic stimulation

\begin{abstract}
A B S T R A C T
Background: Voluntarily opening or closing our eyes results in fundamentally different input patterns and expectancies. Yet it remains unclear how our brains and visual systems adapt to these ocular states. Objective/Hypothesis: We here used transcranial magnetic stimulation (TMS) to probe the excitability of the human visual system with eyes open or closed, in the complete absence of visual inputs.

Methods: Combining Bayesian staircase procedures with computer control of TMS pulse intensity allowed interleaved determination of phosphene thresholds (PT) in both conditions. We measured parieto-occipital EEG baseline activity in several stages to track oscillatory power in the alpha $(8-12 \mathrm{~Hz})$ frequency-band, which has previously been shown to be inversely related to phosphene perception. Results: Since closing the eyes generally increases alpha power, one might have expected a decrease in excitability (higher PT). While we confirmed a rise in alpha power with eyes closed, visual excitability was actually increased (PT was lower) with eyes closed.

Conclusions: This suggests that, aside from oscillatory alpha power, additional neuronal mechanisms influence the excitability of early visual cortex. One of these may involve a more internally oriented mode of brain operation, engaged by closing the eyes. In this state, visual cortex may be more susceptible to top-down inputs, to facilitate for example multisensory integration or imagery/working memory, although alternative explanations remain possible.
\end{abstract}

(c) 2017 Elsevier Inc. All rights reserved.

\section{Introduction}

Most of the time, opening our eyes opens the floodgates of sensation, preparing and allowing us to interact with the environment. Conversely, closing our eyes means that we shut the world out. With closed eyes, we can turn inwards, focusing on our other senses or our thoughts, memories, and mental images. It seems plausible that our brains would have evolved distinct modes of operation for these two ocular states. But it is not immediately clear what exactly this might mean. In the current study, we focused on a brain system very likely to be affected by eye closure; early visual cortex.

\footnotetext{
* Corresponding author. Department of Cognitive Neuroscience, Faculty of Psychology and Neuroscience, Maastricht University, PO Box 616, 6200 MD, Maastricht, The Netherlands.

E-mail address: tom.degraaf@maastrichtuniversity.nl (T.A. de Graaf).

1 Equal contribution.
}

One reflection of brain state is the excitability, or reactivity, of a cortical region. Concerning early visual cortex, a priori one might have opposing hypotheses about the effect of eye closure on visual excitability. Perhaps visual cortex becomes more excitable when opening the eyes, since this act enables the arrival of visual inputs. Conversely, excitability might rather increase with eye closure, since it could be ecologically useful to be sensitive to dark moving shapes even with eyes closed. Approaching the issue from a different perspective, one established consequence of eye closure is an increase in oscillatory power in the alpha $(\sim 10 \mathrm{~Hz})$ frequency band [1]. Moreover, alpha power has been shown to be inversely related to visual excitability [2]. Combining these insights, eye closure could reduce visual excitability. But from a neurocognitive perspective one might again intuit the opposite: Eye closure, by diminishing the likelihood of bottom-up inputs, could free up early visual cortex for top-down inputs, facilitating such faculties as cross-sensory integration, imagery, or working memory (see e.g. Ref. [3]. In sum, the effect of eye closure on visual excitability is not obvious a priori. 
Neuroimaging studies have investigated which brain regions [4-9] and dynamics [3,10-13] behave differently depending on whether our eyes are open or closed. Without any external inputs in either case, these ocular states have been associated with distinct patterns of neural activation. Generally, closing the eyes changed activity in multiple regions of the brain, including early sensory and multisensory regions, while opening the eyes rather increased activity in attentional and oculo-motor regions [3,4,6,7,14-16]. For instance, recent findings by $\mathrm{Xu}$ et al. [17] support two distinct networks underlying these two states, demonstrating increased cross-sensory connectivity and highly integrated information processing for eyes closed, while ascribing a highly specialized mode of information processing to the eyes open state. Following Marx et al. [3], we will refer to these functional states as 'exteroceptive', for eyes open, and 'interoceptive', for eyes closed. The exteroceptive state is characterized by overt attention, while the interoceptive state was associated with multisensory integration, recall of sensory experiences and imagination $[3,6,15,18]$.

Focusing again on early visual cortex, blood oxygen level dependent (BOLD) signal increases when closing the eyes, except for congenitally blind participants [15]. But while such neuroimaging results are interesting, important, and suggestive, they remain at the same time inevitably limited. Non-invasive brain stimulation techniques can provide complementary insights [19-21]. Transcranial magnetic stimulation (TMS) involves magnetic pulses administered at the scalp that enter and excite the brain. Such pulses can disrupt visual processing if applied over occipital cortex at certain delays to a visual stimulus [22-26], but can also induce transient visual experiences called 'phosphenes' [27-30]. The strength of stimulation required to elicit phosphenes on a predetermined proportion of trials (conventionally 0.5 ) is the 'phosphene threshold' (PT): An established measure of cortical excitability of the visual system.

In the current study we directly tested visual excitability under conditions of open versus closed eyes, in the complete absence of visual inputs. We determined PT for both ocular states in a controlled, interleaved, within-subject paradigm, letting a Bayesian staircase algorithm (QUEST, see Methods) control the intensity of TMS pulses on a trial-by-trial basis. Considering the relation between PT and alpha oscillatory power, we placed electroencephalography (EEG) electrodes over parietal and occipital cortex to take into account the classical eye closure effect on alpha power. Looking ahead, parieto-occipital alpha power was indeed higher with closed eyes. At the same time, PT was significantly lower with closed eyes, indicating increased visual excitability.

\section{Methods}

\section{Participants}

Nineteen participants, including two authors (T.G., S.O.), indicated perception of phosphenes and were selected for the experiment. All were screened for TMS safety and provided written informed consent, receiving monetary compensation for participation. Procedures were approved by the local ethics committee.

\section{TMS and EEG parameters}

Single biphasic TMS pulses were applied to occipital cortex using a MC-B70 figure-of-eight coil connected to a MagPro X100 stimulator (MagVenture, Farum, Denmark). The coil handle was oriented laterally to the right, but the exact coil position on the scalp depended on an idiosyncratic phosphene hotspot. In a localization procedure, participants viewed a fixation point on a computer monitor ( $57 \mathrm{~cm}$ viewing distance) while the TMS coil was moved over right occipital regions, administering TMS pulses until participants indicated perceiving a peripheral phosphene in the lower left quadrant relative to fixation. If the reported phosphene retinotopically followed a shift in gaze, the coil location was accepted and fixed by use of a mechanical arm. During the main experiment, TMS intensity was variable, determined per trial by the staircase procedure (see below). A test range was enforced on the staircase algorithm, however, to the effect that pulses were maximally at $70 \%$ of maximum stimulator output, with a lower bound adapted from $25 \%$ to $20 \%$ to finally $10 \%$, as we noted that some participants' PTs were lower than originally anticipated.

We applied four EEG electrodes to locations on the international 10-20 coordinate system [31], attaching them using conductive gel $\left(\right.$ Ten $20^{\circledR}$, DO Weaver, Aurora, CO, USA) and leading into a headbox connected to BrainVision amplifiers (BrainProducts $\mathrm{GmbH}$, Munich, Germany). A parietal electrode was placed at P3, an occipital electrode was placed at 01 , a ground to $\mathrm{Cz}$ and a reference electrode to the left mastoid. No EEG data were recorded for one participant due to time constraints, resulting in 18 full EEG datasets. In a subsample of participants $(\mathrm{N}=9)$ electrooculography (EOG) was also acquired, with two electrodes (HEOG/VEOG) attached near the left eye. EEG/ EOG signals were recorded using VisionRecorder software (BrainProducts $\mathrm{GmbH}$, Munich, Germany), with a sampling frequency of $2500 \mathrm{~Hz}$, a notch filter at $50 \mathrm{~Hz}$, and filtered with high and low cutoff values of $250 \mathrm{~Hz}(100 \mathrm{~Hz}$ for EOG channels) and $0.1 \mathrm{~Hz}$, respectively.

\section{Design and task}

The experimental session contained several stages, after application of EEG electrodes and fixation of the TMS coil. We asked participants to relax for $90 \mathrm{~s}$ while we acquired baseline EEG signals at different moments throughout the experimental session. We also determined phosphene thresholds (PT) several times under different conditions. These conditions included wearing a blindfold or not, with opened or closed eyes. If not wearing the blindfold, participants were looking at a fixation cross on a computer monitor in a darkened but not pitchblack room. The blindfold (Mindfold Inc., Tucson, AZ) was used to create a condition of absolute darkness. It is a fully darkening mask that does not directly touch the eyes, allowing participants to comfortably open or close their eyes underneath. Once participants were wearing the blindfold, we switched the lights in the room fully on or off and asked whether participants could tell the difference, which they reportedly could not. One participant did report a shift in the blindfold during the experiment so that some light penetrated the blindfold for part of the measurement, this participant was marked as having Grounds for Exclusion (GfE; see below). Though the blindfold in all other cases provided perfect darkness, redundantly we nevertheless turned off the lights in the room during the measurements. Under these various conditions, the full sequence of measurements was as follows:

\footnotetext{
- baseline EEG, eyes open without blindfold (normalization target 'EEGstart')

- PT staircase, eyes open without blindfold (normalization target 'PTstart')

(participant starts wearing the blindfold)

- baseline EEG 'EEG pre', eyes open (or closed)

- baseline EEG 'EEG pre', eyes closed (or open)

- interleaved PT staircases for eyes open and closed

- baseline EEG 'EEG post', eyes closed (or open)

- baseline EEG 'EEG post', eyes open (or closed)

(participant stops wearing the blindfold)
} 
- PT staircase, eyes open without blindfold (normalization target 'PTend')

- baseline EEG, eyes open without blindfold (normalization target 'EEGend')

As detailed below, the EEG baselines and PTs obtained without the blindfold at the start and end of each experimental session (PTstart/PTend, EEGstart/EEGend) served as normalization targets for the data of interest. The data of interest were the phosphene thresholds and EEG measures for eyes open and closed in darkness while wearing the blindfold.

Across participants, there were two possible orderings of the EEG baseline measurements with eyes open or closed after putting the blindfold on; it was either open > closed > interleaved PT block $>$ closed $>$ open $(\mathrm{N}=8)$, or alternatively, closed $>$ open $>$ interleaved PT block $>$ open $>$ closed $(\mathrm{N}=10)$.

The core measurement in this study was the block of interleaved PT determination for eyes open and closed in darkness. Based on an auditory instruction 'open' or 'close', presented between 3 and $4 \mathrm{~s}$ (random) prior to each TMS pulse, participants opened or closed their eyes on a trial-by-trial basis. In the baseline PT staircases without blindfold, with eyes always open, they always received the instruction 'open' prior to each TMS pulse. In all PT staircase procedures, the task was to indicate, whenever ready, by keyboard button presses using index versus middle fingers of the right hand whether a phosphene had been perceived or not.

\section{PT staircase procedure}

The TMS stimulator was triggered by a stimulus PC through a parallel port, and was instructed by the same PC through serial port communication prior to each pulse to set a given TMS intensity for the next trial. These processes, as well as presentation of visual fixation crosses and auditory cues, were managed through Presentation software (NeuroBehavioral Systems, Albany, CA). Presentation interfaced with Matlab software to use a Bayesian staircase algorithm implemented in Psychtoolbox; QUEST [32]. This staircase algorithm takes certain priors (see below) and then continually updates its threshold estimations based on the total pattern of responses (in this case phosphene-yes versus phosphene-no) for the tested stimulus intensities (in this case percentage TMS stimulator output). One benefit of this algorithm is that it advises the user on each next test value, ensuring a high sampling density at the most relevant section of the psychometric curve (in this case phosphene perception versus TMS intensity),

Excitability Gain $=(($ PTopen - PTclosed $) /$ mean $($ PTstart, PTend $)){ }^{*} 100$
3.5, gamma 0.01 , delta 0.01 . The aim performance was 0.5 (50\% phosphene report) and the number of trials per staircase was 40 . Time between TMS pulses was jittered around $6 \mathrm{~s}( \pm 1 \mathrm{~s})$.

\section{Analysis}

We focused the analyses on the data acquired while wearing the blindfold, although we will also report PT estimates without blindfold. We accounted for individual baseline differences in both EEG measures and PTs while wearing the blindfold, by normalizing them to EEG measures and PTs obtained without blindfold at the beginning and end of each experimental session (the 'normalization targets'; see procedure above and normalization description below). As mentioned, one subject reported penetration of some light into the blindfold due to a shift in the mask and therefore had Grounds for Exclusion (GfE). Four other participants had GfE because the validity of final PT estimates from their staircase procedures could be contested, based on repeated trials at upper or lower boundaries of the test range. Concretely, we marked participants as having GfE if they were 'stuck' at one boundary of the test range consecutively for minimally $25 \%$ of trials, in either the eyes open or eyes closed conditions. For the sake of transparency, all data and analyses are reported for the subject sample both including and excluding the GfE participants, and the progression of test values (i.e. staircases) can be inspected in Supplementary Material for all participants. As will become clear; in- or excluding GfE participants led to similar results.

We performed two analyses on the behavioral data, which consisted of 'yes' (phosphene perceived) and 'no' (phosphene not perceived) responses to single TMS pulses over a range of intensities.

1) We extracted the intensity leading to $50 \%$ phosphene perception as estimated by the Bayesian staircase algorithm (QuestMean function), per condition per participant. These non-normalized PTs for eyes open (PTopen) and eyes closed (PTclosed) were directly compared in a two-tailed paired-samples $t$-test.

2) We calculated the Excitability Gain per participant to reflect the decrease in PT for eye closure, normalized to individual PT baselines while not wearing the blindfold (see procedure above). Concretely, this involved a) subtracting PTclosed from PTopen, b) normalizing this difference to the mean PT of PTstart/ PTend, and c) multiplying this by 100 , to express eye closure induced excitability gain relative to individual baseline as a percentage. All this is summarized in the formula: which depends on the requested level of performance (in this case $50 \%$ perceived phosphenes).

In the interleaved PT block, we maintained separate staircases for the eyes open condition and the eyes closed condition with pseudorandomly interleaved trials. Within the test range (see above), we adopted the suggested next TMS pulse intensity level, rounding the suggested percentage of maximal machine output to integers. We requested QuestQuantile for next test values, based final threshold estimates on QuestMean, and supplied the following parameters to the staircase: prior mean $\log 10(50)$, prior sd 1 , beta
We tested mean Excitability Gain against zero in a one-sample ttest.

\section{Analysis of EEG data}

We recorded EEG data both in dedicated EEG baseline measurements of $\sim 90 \mathrm{~s}$, and during the TMS PT staircase procedures. All data were analyzed in Matlab using the Fieldtrip toolbox [33]. 
The EEG baseline segments were cut into epochs of $1000 \mathrm{~ms}$, which were subjected to a Fast Fourier Transform (FFT) to obtain spectral profiles. These were then averaged per participant per condition (eyes open or eyes closed). The spectral power of frequency bins in the alpha range $(8-12 \mathrm{~Hz}$ ) was summed per channel, leading to our scalar measure of 'alpha power'. These alpha power measures (AP) were then normalized and expressed as a relative change to analogous AP measures extracted from the EEG baselines obtained at the start ('EEGstart') and end ('EEGend') of each experimental session (see procedure above):

Normalized $A P=(A P-$ mean $(A P$ EEGstart, AP EEGend $)) /$ mean $(A P$ EEGstart, AP EEGend).

We did this separately for the EEG baselines acquired just prior to the interleaved PT staircase procedures ('EEGpre') and just afterwards ('EEGpost'), to allow a statistical evaluation of factor 'prepost'.

We also measured EEG and extracted alpha power during the interleaved PT staircase procedure. We extracted epochs of $1000 \mathrm{~ms}$ just prior to TMS pulses (1050-50 ms before pulse), since we were most interested in alpha power just before the induction of phosphenes but uncontaminated by the TMS-induced artifact. These epochs were analyzed identically to the baseline AP data and again normalized to the EEGstart/EEGend baseline AP. Statistically, AP was compared between conditions in standard repeated-measures ANOVAs (using SPSS software, IBM, Armonk, NY, USA) and followup tests as indicated in the Results section. In ANOVAs, Greenhouse-Geisser correction was applied if Mauchley's test suggested non-sphericity. We hypothesized that closing the eyes should lead to higher alpha power in EEG.

We also acquired EOG measures from a subsample of participants $(\mathrm{N}=9)$. Given this small sample size and the practical limitation of having the electrode cable running underneath and pressured by the blindfold, we will not interpret these findings in depth. To obtain a first indication of whether or not eye movements are of interest to future studies, we calculated from the same $1000 \mathrm{~ms}$ epochs prior to TMS pulses the variance in both the HEOG/ VEOG channels and compared them between eyes closed and eyes open trials (results in Supplementary Material).

On a final note; for all analyses of normalized PT, EEG, and EOG data, we always performed outlier removal based on the same criteria. Within participants and conditions, EEG epochs with signal variance 3 standard deviations above mean variance were removed prior to further analyses. In following group analyses, participants with normalized measures (PT, alpha power, EOG variance) minimally 3 standard deviations above or below the group mean in minimally one condition in an analysis were excluded from that particular analysis. Note that the occurrence of such exclusions is transparent per statistical test by looking at the reported degrees of freedom.

\section{Results}

We obtained phosphene thresholds (PTs) from all staircases with eyes open/closed and blindfold on/off and present them in Table 1. Note that while the group means of PT baselines PTstart/ PTend (the baseline measures at beginning and end of each session) were highly similar and not statistically different $(t(18)=0.36$, $\mathrm{p}=0.73$ ), for individual participants these PTs could vary substantially, for a variety of possible reasons to which we return in the Discussion. For the moment, this variability strongly emphasizes the importance of having interleaved the staircases for the main conditions of interest: PTs for eyes open versus eyes closed without visual inputs (i.e. while wearing the blindfold).
Phosphene thresholds for eyes open and closed in the absence of visual inputs

The Bayesian Quest staircase procedure threshold estimations resulted in higher PTs for the eyes open condition $(\mathrm{M}=38.0$, $\mathrm{SD}=16.2)$ as compared to the eyes closed condition $(\mathrm{M}=35.9$, $\mathrm{SD}=14.7$ ) including all participants. A two-sided paired-samples $t$ test revealed a statistically significant difference $(t[18]=3.03$, $p=0.007)$. Differences were even more pronounced when excluding participants with Grounds for Exclusion (GfE) (PTopen $\mathrm{M}=35.9, \mathrm{SD}=11.9$, PTclosed $\mathrm{M}=33.6, \mathrm{SD}=10.8, t[13]=4.17$, $p=0.001$ ).

A good representation of this effect is the Excitability Gain (decrease in PT with eye closure weighed by inherent visual excitability, see Methods), displayed for all participants in Fig. 1. GfE participants are shaded light grey. This figure makes clear that there was a highly consistent positive Excitability Gain, indicating a relative increase in visual excitability upon closing the eyes, confirmed statistically in one-sample t-tests including $(\mathrm{M}=4.7$, $\mathrm{SD}=5.9, t(18)=3.49, p=0.003)$ and excluding GfE participants $(\mathrm{M}=5.7, \mathrm{SD}=5.2, \mathrm{t}(13)=4.07, \mathrm{p}=0.001)$. Fig. $\mathrm{S} 1$ in Supplementary Material shows the progression of test intensities in individual staircases, which provides only an indirect reflection of visual excitability but demonstrates nicely that the staircases neatly converged to PTs for all participants not marked GfE.

In sum, closing the eyes led to higher cortical excitability, reflected by lower phosphene thresholds, as compared to opening the eyes.

\section{Alpha power for eyes open and closed in the absence of visual inputs}

Phosphene thresholds have previously been related to parietooccipital oscillatory alpha power, which was inversely related to phosphene perception on a trial-by-trial basis [2]. If alpha power were the only determinant of PT, then based on this we should expect lower alpha power for eyes closed since we observed a lower PT. Yet, conventionally, eye closure has rather been related to increased alpha power [1]. This discrepancy made it crucial to evaluate whether in our participants, and during our tasks, parieto-

Table 1

Phosphene thresholds at the start and end without blindfold, and with eyes open and closed in darkness from an interleaved staircase procedure.

\begin{tabular}{lllll}
\hline & PTstart & PTopen & PTclosed & PTend \\
\hline S1 & 33.7 & 42.1 & 38.9 & 43.5 \\
S2 *GfE & 34.0 & 21.7 & 21.3 & 23.8 \\
S3 & 39.1 & 36.5 & 33.3 & 38.7 \\
S4 & 36.1 & 42.4 & 41.4 & 36.6 \\
S5 & 41.7 & 36.8 & 32.4 & 36.7 \\
S6 & 45.7 & 45.4 & 42.4 & 38.2 \\
S7 & 31.1 & 38.8 & 35.7 & 36.7 \\
S8 ${ }^{*}$ GfE & 45.1 & 57.7 & 60.7 & 46.5 \\
S9 & 34.7 & 23.9 & 21.9 & 26.1 \\
S10 ${ }^{*}$ GfE & 22.6 & 20.2 & 19.7 & 21.8 \\
S11 ${ }^{*}$ GfE & 68.7 & 80.6 & 70.3 & 79.6 \\
S12 & 43.2 & 31.3 & 33.8 & 30.5 \\
S13 & 16.9 & 16.5 & 17.1 & 16.7 \\
S14 & 25.2 & 26.9 & 23.7 & 29.7 \\
S15 & 32.1 & 20.7 & 19.6 & 30.5 \\
S16 & 40.1 & 45.7 & 42.8 & 41.3 \\
S17 *GfE & 36.6 & 39.2 & 39.6 & 41.1 \\
S18 & 38.1 & 32.5 & 30.8 & 31.8 \\
S19 & 56.0 & 62.4 & 57.2 & 60.9 \\
\hline mean & $\mathbf{3 7 . 9}$ & $\mathbf{3 8 . 0}$ & $\mathbf{3 5 . 9}$ & $\mathbf{3 7 . 4}$ \\
mean noGfE & 36.7 & 35.9 & 33.6 & 35.6 \\
\hline & & & & \\
\hline & & & & \\
\hline
\end{tabular}




\section{Excitability Gain from eye closure}

20

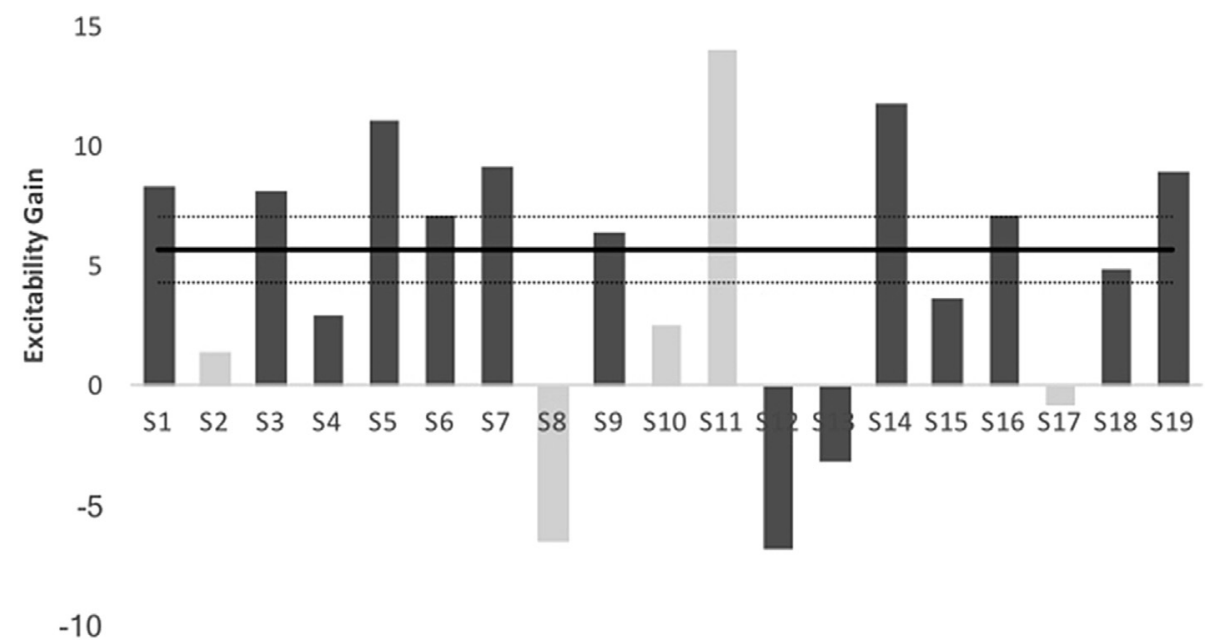

Fig. 1. Excitability Gain.

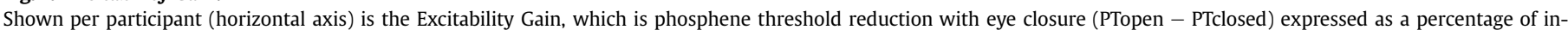

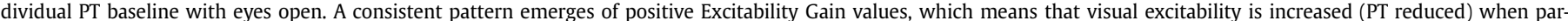

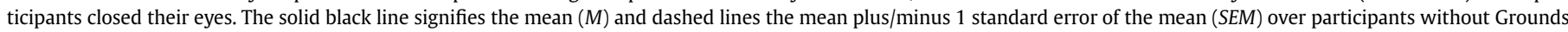

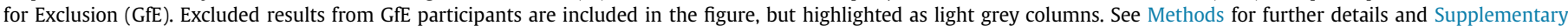
Fig. S1 for progression of all individual staircases.

occipital alpha power did not unexpectedly decrease, rather than increase, with eye closure.

The critical baseline measurements of alpha power, using EEG at rest and normalized to independent individual baselines (see Methods), were obtained for two electrodes (P3 and 01; factor 'channel') both immediately prior to and immediately after the interleaved PT staircase block (factor 'pre-post'), while participants were wearing the blindfold and keeping their eyes closed or open (factor 'eyes') for prolonged periods of rest (90 s). The various analyses reported below revealed main effects of channel, with 01 yielding higher alpha power, but we do not report this in more detail.

Excluding the participant who reported light inside the blindfold and one outlier participant (Methods), we performed a pre- post $\mathrm{x}$ open-closed $\mathrm{x}$ channel repeated-measures ANOVA. This revealed an unexpected interaction between pre-post $\mathrm{x}$ openclosed $(F(1,15)=7.02, p=0.018)$, suggesting that eye closure affected alpha power, but differentially for the EEG baselines acquired before versus after the phosphene threshold staircases procedure. As illustrated in Fig. 2A, normalized alpha power was significantly different for the pre-staircase EEG baseline measurement 'EEGpre' (open-closed x channel RM-ANOVA, factor openclosed $F(1,15)=24.78, p<0.001$ ), so briefly after participants put on the blindfold, but not in the post-staircase baseline measurement 'EEGpost' $(\mathrm{F}(1,15)=0.05, \mathrm{p}=0.835)$.

This observation makes it relevant to determine what effect eye closure had on alpha power during the PT staircase procedures. After all, if eye closure during the staircases actually decreased

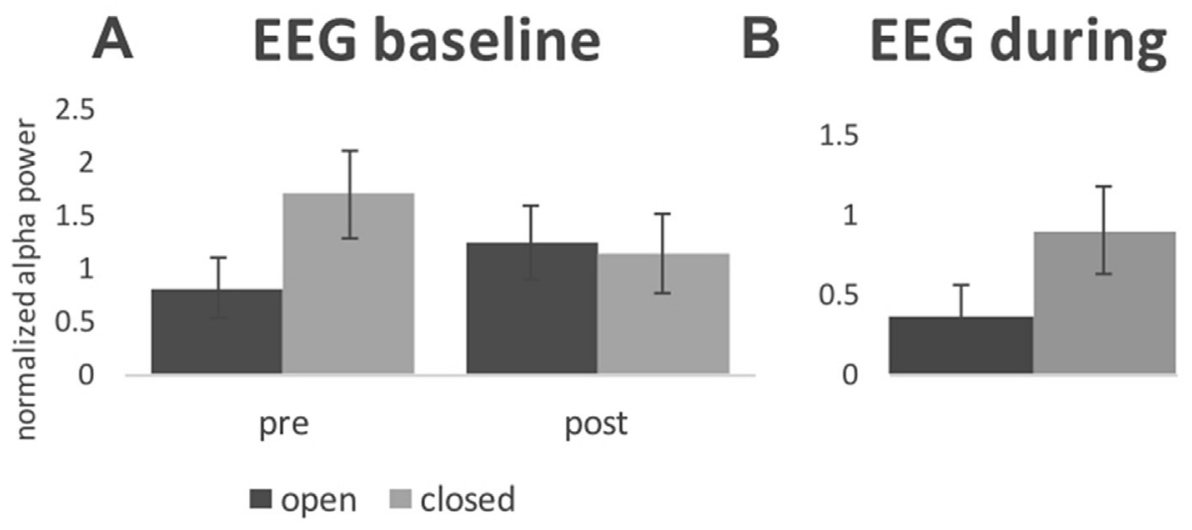

Fig. 2. Normalized alpha power.

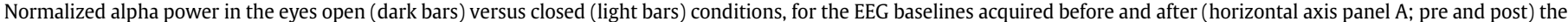

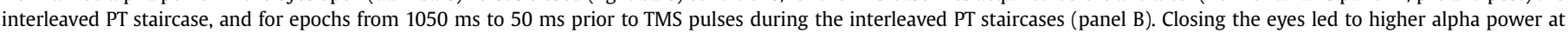
electrode positions P3/01, at rest before, as well as during, but no longer after the interleaved PT staircases. 
alpha power, instead of increasing it as hypothesized, this could explain the PT results. But as shown in Fig. 2B, this was not the case. In fact, during the staircases there was significantly higher normalized alpha power in eyes closed trials as compared to eyes open trials (open-closed $\mathrm{x}$ channel RM-ANOVA, main effect of open-closed: $F(1,16)=11.18, p=0.004)$.

In the Supplementary Material, we report whether the eye closure effect on alpha power decreased over the course of the interleaved PT staircases procedure; there appeared to be such a pattern but it was not statistically supported. See also Supplementary Material for results of the EOG analysis. Briefly; no clear condition effects were found but here sample size and interpretability were limited. We thus found no indication that differences in eye movements caused the observed effects, but it is noteworthy that previous work found that lateral eye movements are more frequent with eyes open [34], at least in a normal perceptual state without a blindfold.

To summarize, both at rest and during the PT staircase procedure, alpha power was significantly higher with eyes closed as compared to eyes open, even in the complete absence of visual inputs. The baseline measurement at the end of the session no longer showed this effect, but importantly did not suggest an inverse effect either, which is most relevant in the current context. Given the previously established inverse relation between alpha power and occipital excitability measured by phosphene $[2,35]$ or visual [36] perception, the mechanism behind higher visual excitability with eyes closed needs to be sought elsewhere.

\section{Discussion}

In the current study we asked how the visual system of the brain reacts to the act of closing or opening the eyes. More specifically, we evaluated cortical excitability of early visual cortex in the absence of visual inputs through the determination of phosphene thresholds (PTs). These PTs were determined in individual participants with Bayesian adaptive algorithms (see Methods), and interleaved for the relevant conditions of opening and closing the eyes. Lastly we measured resting state EEG under similar conditions, and measured EEG concurrently with the PT staircase procedure, to evaluate whether any potential changes in PT could be explained by changes in oscillatory power in the alpha frequency band.

The main observation in the current dataset was that PTs were lower in the eyes closed condition. This suggests that early visual cortex becomes more responsive to direct stimulation with TMS upon closing the eyes. Critically, the interleaved determination of PTs ensured that the effect of ocular state on PTs was not confounded by other factors that may influence cortical excitability, such as dark adaptation or global changes of arousal. Interestingly, a different approach to probe visual sensitivity, using intra-oral stimulation of the retina in total darkness to elicit visual experiences [37], actually revealed higher visual detection thresholds with eyes closed $[38,39]$. This opposite finding is instructive, given the difference in stimulation methods: retinal stimulation versus direct cortical stimulation. For instance; phosphenes elicited by electrical stimulation of the retina, but not magnetic stimulation of occipital cortex, are reduced by visual suppression through saccades [40]. Distinctions like these demonstrate the unique contribution of phosphene measurements to the study of the human visual system.

Toscani et al. [38] related their decreased visual sensitivity upon eye closure, to eye closure induced increases of alpha power. This classic 'Berger effect' is well established if opening the eyes also involves imminent visual inputs, but may be less prominent in total darkness (e.g. Refs. [41,42]). However, even in total darkness alpha power has been reported to increase with eye closure (e.g.
Refs. [43-45], as we replicated here. In our experiment, this alpha effect unexpectedly disappeared in the post-staircase baseline measurement. But in fact this disappearance seems to have been noticed in the very earliest reports on alpha power across eye closure conditions; hinting that the 'Berger effect' is not necessarily stable but disappears after some time in darkness [41] in Ref. [46]. Also, alpha desynchronization by auditory stimuli diminishes with repeated stimulation [47], and alpha power is related to wakefulness/vigilance, with drowsiness apparently diminishing the Berger effect [45].

Such findings should remind us that many factors affect brain state, and that assuming a stable brain state is likely an oversimplification. This imposes limitations on many experiments, including this one, which in fact implicitly or explicitly assume a stable brain state across multiple trials of one experimental condition. In our study, the phosphene threshold staircase procedure, for both eyes open and closed conditions, assumes a stable threshold that it attempts to estimate, and the final outcome reflects responses to test values over the full (approximately $8 \mathrm{~min}$ ) duration of the staircase procedure. The fact that the Berger effect was not obtained in the post-staircase EEG baseline, but was clear in the pre-staircase EEG baseline, could suggest that brain state was, also in our study, not stable. In that sense, it was important to on the one hand interleave the trials of both conditions, and on the other hand evaluate alpha power during the staircase procedure. The Berger effect was significant during the staircases (Fig. 2B), and there was insufficient statistical power or support for a change in Berger effect over the course of the staircase procedure (Fig. S2).

In any case note that our main findings would have remained of interest even in the case of a full disappearance of the Berger effect during the staircase procedures, since a lack of Berger effect would still not have explained the lower PT with eyes closed in any way. For the current study it was primarily relevant that we did not observe an "inverse Berger effect" (higher alpha power with eyes open), given previous studies relating alpha power to phosphene perception (in fact, also alpha phase: [48]. Specifically, Romei and colleagues showed that higher alpha power is associated with a lower probability of inducing a phosphene across subjects [35] and trials [2]. At the same time, we would like to emphasize that our results are not construed to be in conflict with those findings. The studies are too different in design, methodology, and scope, to directly compare. Also, the role of alpha oscillations in visual excitability is not disputed. Posterior alpha activity is thought to reflect inhibitory processing in the brain [49,50], and a long line of research has shown that alpha activity increases in areas not relevant for the current task, effectively reducing the responsiveness (or excitability) of these brain areas.

Other work that relates to but is not immediately comparable to the current study, concerns the effects of light deprivation on cortical excitability. TMS has been used to assess cortical excitability in complete darkness [51-53], generally revealing decreased PTs after several tens of minutes, hours, or even days of light deprivation. It is thus conceivable that the relatively short exposure to complete darkness in our study ( $15 \mathrm{~min})$ had a similar effect on cortical excitability, although we are not aware of evidence for light deprivation effects on such a short time-scale. Also, PTs with eyes open at the beginning of the session, during blindfolding, and after re-exposure to light at the end of the session did not appreciably differ from each other (see Table 1). Lastly, the interleaved staircases ensured that any orthogonal variable affecting brain state would have affected PT results in both eye conditions similarly.

Phosphene thresholds directly reflect the excitability of visual cortex [29,54]. Our PT results, taken together with previous research, lead to an interesting conclusion. Since light deprivation or the known relationship between alpha power and phosphene 
perception cannot explain our PT results, an additional mechanism may affect visual excitability upon closing the eyes, which could mean that alpha power is not the only determinant of PT. This alternative mechanism may be part of a cascade of processes in the human brain, initiated by the act of closing the eyes, that results in a more internal mode of operation $[3,18]$.

FMRI studies previously showed an increase in BOLD signals in early visual regions upon closing the eyes $[3,6,15,18]$. Though the complex relation between BOLD signals and neural activity (especially in the absence of stimulus drives) rendered the effect of eye closure on visual excitability an empirical question, the decrease of PTs we observed seems in line with these fMRI studies. Our results complement this work, by providing additional information and possible interpretations. One such interpretation is that early visual regions are 'more engaged' with closed eyes in the absence of visual inputs, in what has been called the 'interoceptive mode' of brain operation $[3,6,15,18]$.

Going beyond early visual cortex alone, recent evidence suggests that the 'interoceptive mode' is characterized by a strong coupling of the visual system with motor, somatosensory and auditory systems, possibly indicating high synchronization across sensory modalities with eyes closed [17]. This could improve phosphene perception as TMS pulses can inherently be described as multisensory events (including somatosensory and auditory effects). Increased connectivity among sensory cortices could therefore boost multisensory processes that decrease detection thresholds in spite of alpha power increases [55,56]. In contrast, with eyes open the sensory modalities except for vision seem to be suppressed to allocate more energy for exteroceptive processing $[4,16]$ thereby reducing the impact other senses might have on phosphene elicitation. On a general level, this network perspective is further supported by recent findings that perception of nearthreshold stimuli in the auditory and somatosensory domain depends on the connectivity state of distributed networks, to some extent irrespective of localized oscillatory activity [57,58].

Increased connectivity to early visual cortex with closed eyes could also be relevant for higher cognitive processes. Since the lingual gyrus and the fusiform gyrus as part of the visual system are considered to be important for visual imagery and memory [59], the strong connections with other sensory modalities with eyes closed may lead to imagination and recall of perceptual experiences [3]. Imagination has been suggested to involve the same brain structures and modalities which are activated in actual perception [60]. Visual mental imagery was found to be impaired by repetitive TMS over the medial occipital cortex as compared to a sham condition [61]. Interestingly, Sparing et al. [62] reported decreased PT during a visual imagery task in blindfolded subjects, compared to baseline PT, while no such modulation was found during an auditory control task (see also [63].

\section{Methodological implications}

We acquired PTs with eyes open and closed in complete darkness, but also PTs with eyes open without a blindfold, while staring at a computer monitor in a dimmed laboratory setting, once at the start and again at the end of the experimental session. As shown in Table 1, these two baseline PTs could differ on the individual level, but apparently not in any consistent direction (no significant difference on the group level). It thus appears that there are different sources of variability across the experimental session both to the effect of increasing PT and of decreasing PT, to which different participants are idiosyncratically sensitive. One could consider individual differences in dark adaptation, arousal (is the participant nervous about TMS in the beginning of the experiment of not?), fatigue/boredom, changes in response criteria for phosphene yes/ no or task strategies, small head movements leading to a shift in effective TMS coil position, to name just a few. The variability between PTs observed, in combination with these considerations, leads to the conclusion that interleaved staircases may be crucial to obtain measures of PT in a setting such as this. The difference between PT with eyes open and closed while wearing the blindfold was, in context of the variability discussed above, relatively small. Yet it was very consistent, perhaps exactly because we controlled for all these sources of variability by implementing interleaved staircases, made possible by algorithmic control of TMS intensity per trial.

\section{Conclusion}

The effect of ocular state on visual excitability reported here provides an interesting new insight into the various brain mechanisms engaged by simply closing or opening our eyes. There are still several possible explanations, ranging from ecological to low-level (e.g. multisensory enhancement) to high-level (e.g. early visual cortex engagement for top-down/working memory/imagery) proposals. Overall it appears that visual excitability is determined by multiple processes, in which alpha activity is one but not alone.

\section{Acknowledgements}

Alexander T. Sack, Tom A. de Graaf, and Sanne ten Oever were supported by The Netherlands Organization for Scientific Research (NWO; 453-15-008, 451-13-024, and 406-11-068). Felix Duecker was supported by the European Union's Horizon 2020 research and innovation programme under the Marie Skłodowska-Curie Grant Agreement No. 708492. Yuliya Stankevich was supported by Deutsche Forschungsgemeinschaft (DFG) by the Collaborative Research Centre 940: "Volition and Cognitive Control: Mechanisms, Modulators, Dysfunctions".

\section{Appendix A. Supplementary data}

Supplementary data related to this article can be found at http:// dx.doi.org/10.1016/j.brs.2017.04.127.

\section{References}

[1] Berger H. Über das Elektroenkephalogramm des Menschen. Arch Psychiatr Nervenkr 1929;87:527-70.

[2] Romei V, Brodbeck V, Michel C, Amedi A, Pascual-Leone A, Thut G. Spontaneous fluctuations in posterior alpha-band EEG activity reflect variability in excitability of human visual areas. Cereb Cortex 2008;18(9):2010-8.

[3] Marx E, Stephan T, Nolte A, Deutschländer A, Seelos KC, Dieterich M, et al. Eye closure in darkness animates sensory systems. Neuroimage 2003;19(3): 924-34.

[4] Bianciardi M, Fukunaga M, van Gelderen P, Horovitz SG, de Zwart JA, Duyn JH. Modulation of spontaneous fMRI activity in human visual cortex by behavioral state. Neuroimage 2009;45(1):160-8.

[5] Liu D, Dong Z, Zuo X, Wang J, Zang Y. Eyes-open/eyes-closed dataset sharing for reproducibility evaluation of resting state fMRI data analysis methods. Neuroinformatics 2013;11(4):469-76.

[6] Marx E, Deutschländer A, Stephan T, Dieterich M, Wiesmann M, Brandt T. Eyes open and eyes closed as rest conditions: impact on brain activation patterns. Neuroimage 2004;21(4):1818-24.

[7] McAvoy M, Larson-Prior L, Nolan TS, Vaishnavi SN, Raichle ME. d'Avossa G. Resting states affect spontaneous BOLD oscillations in sensory and paralimbic cortex. J Neurophysiol 2008;100(2):922-31.

[8] Yang H, Long X-Y, Yang Y, Yan H, Zhu C-Z, Zhou X-P, et al. Amplitude of low frequency fluctuation within visual areas revealed by resting-state functional MRI. Neuroimage 2007;36(1):144-52.

[9] Zang Y-F, He Y, Zhu C-Z, Cao Q-J, Sui M-Q, Liang M, et al. Altered baseline brain activity in children with ADHD revealed by resting-state functional MRI. Brain Dev 2007;29(2):83-91.

[10] Patriat R, Molloy EK, Meier TB, Kirk GR, Nair VA, Meyerand ME, et al. The effect of resting condition on resting-state fMRI reliability and consistency: a comparison between resting with eyes open, closed, and fixated. Neuroimage 2013;78:463-73. 
[11] Van Dijk KRA, Hedden T, Venkataraman A, Evans KC, Lazar SW, Buckner RL. Intrinsic functional connectivity as a tool for human connectomics: theory, properties, and optimization. J Neurophysiol 2010;103(1):297-321.

[12] Yan C, Liu D, He Y, Zou Q, Zhu C, Zuo X, et al. Spontaneous brain activity in the default mode network is sensitive to different resting-state conditions with limited cognitive load. PLoS One 2009;4(5):e5743.

[13] Zou Q, Long X, Zuo X, Yan C, Zhu C, Yang Y, et al. Functional connectivity between the thalamus and visual cortex under eyes closed and eyes open conditions: a resting-state fMRI study. Hum Brain Mapp 2009;30(9):3066-78.

[14] Hüfner K, Stephan T, Glasauer S, Kalla R, Riedel E, Deutschländer A, et al. Differences in saccade-evoked brain activation patterns with eyes open or eyes closed in complete darkness. Exp Brain Res 2008;186(3):419-30.

[15] Hüfner K, Stephan T, Flanagin VL, Deutschländer A, Stein A, Kalla R, et al. Differential effects of eyes open or closed in darkness on brain activation patterns in blind subjects. Neurosci Lett 2009;466(1):30-4.

[16] Niven JE, Laughlin SB. Energy limitation as a selective pressure on the evolution of sensory systems. J Exp Biol 2008;211(Pt 11):1792-804.

[17] Xu P, Huang R, Wang J, Van Dam NT, Xie T, Dong Z, et al. Different topological organization of human brain functional networks with eyes open versus eyes closed. Neuroimage 2014;90:246-55.

[18] Wiesmann M, Kopietz R, Albrecht J, Linn J, Reime U, Kara E, et al. Eye closure in darkness animates olfactory and gustatory cortical areas. Neuroimage 2006;32(1):293-300.

[19] Hallett M. Transcranial magnetic stimulation: a primer. Neuron 2007;55(2): 187-99.

[20] Pascual-Leone A, Walsh V, Rothwell J. Transcranial magnetic stimulation in cognitive neuroscience-virtual lesion, chronometry, and functional connectivity. Curr Opin Neurobiol 2000;10(2):232-7.

[21] Sack AT. Transcranial magnetic stimulation, causal structure-function mapping and networks of functional relevance. Curr Opin Neurobiol 2006;16(5): 593-9.

[22] Amassian VE, Cracco RQ, Maccabee PJ, Cracco JB, Rudell A, Eberle L. Suppression of visual perception by magnetic coil stimulation of human occipital cortex. Electroencephalogr Clin Neurophysiol 1989;74(6):458-62.

[23] Beckers G, Hömberg V. Impairment of visual perception and visual short term memory scanning by transcranial magnetic stimulation of occipital cortex. Exp Brain Res 1991;87(2):421-32.

[24] Corthout E, Uttl B, Walsh V, Hallett M, Cowey A. Timing of activity in early visual cortex as revealed by transcranial magnetic stimulation. Neuroreport 1999;10(12):2631.

[25] Kammer T. Masking visual stimuli by transcranial magnetic stimulation. Psychol Res 2007;71(6):659-66.

[26] Graaf TA, Koivisto M, Jacobs C, Sack AT. The chronometry of visual perception: review of occipital TMS masking studies. Neurosci Biobehav Rev 2014;45: 295-304

[27] Barker AT, Jalinous R, Freeston IL. Non-invasive magnetic stimulation of human motor cortex. Lancet 1985;1(8437):1106-7.

[28] Kammer T. Phosphenes and transient scotomas induced by magnetic stimulation of the occipital lobe: their topographic relationship. Neuropsychologia 1999;37(2):191-8.

[29] Marg E, Rudiak D. Phosphenes induced by magnetic stimulation over the occipital brain: description and probable site of stimulation. Optom Vis Sci 1994;71(5):301-11.

[30] Meyer BU, Diehl R, Steinmetz H, Britton TC, Benecke R. Magnetic stimuli applied over motor and visual cortex: influence of coil position and field polarity on motor responses, phosphenes, and eye movements. Electroencephalogr Clin Neurophysiol Suppl 1991;43:121-34.

[31] Klem GH, Lüders HO, Jasper HH, Elger C. The ten-twenty electrode system of the international federation. The international federation of clinical neurophysiology. Electroencephalogr Clin Neurophysiol Suppl 1999;52:3-6.

[32] Watson AB, Pelli DG. QUEST: a Bayesian adaptive psychometric method. Percept Psychophys 1983:33(2):113-20.

[33] Oostenveld R, Fries P, Maris E, Schoffelen J-M. FieldTrip: open source software for advanced analysis of MEG, EEG, and invasive electrophysiological data. Comput Intell Neurosci 2011;2011:156869.

[34] Takeda M, Yoshimura H. Lateral eye movement while eyes are closed. Percept Mot Ski 1979;48(3 Pt 2):1227-31.

[35] Romei V, Rihs T, Brodbeck V, Thut G. Resting electroencephalogram alphapower over posterior sites indexes baseline visual cortex excitability. Neuroreport 2008;19(2):203-8.

[36] Lange J, Oostenveld R, Fries P. Reduced occipital alpha power indexes enhanced excitability rather than improved visual perception. J Neurosci 2013;33(7):3212-20.

[37] Volkmann FC, Riggs LA, Moore RK. Eyeblinks and visual suppression. Science 1980;207(4433):900-2.
[38] Toscani M, Marzi T, Righi S, Viggiano MP, Baldassi S. Alpha waves: a neural signature of visual suppression. Exp Brain Res 2010;207(3-4):213-9.

[39] Volkmann FC, Riggs LA, Ellicott AG, Moore RK. Measurements of visual suppression during opening, closing and blinking of the eyes. Vis Res 1982;22(8): 991-6.

[40] Thilo KV, Santoro L, Walsh V, Blakemore C. The site of saccadic suppression. Nat Neurosci 2004;7(1):13-4.

[41] Adrian ED, Matthews BHC. The Berger Rhythm: potential changes from the occipital lobes in man. Brain 2010:133(Pt 1):3-6.

[42] Bohdanecký Z, Indra M, Lánský P, Radil-Weiss T. Alternation of EEG alpha and non-alpha periods does not differ in open and closed eye condition in darkness. Acta Neurobiol Exp (Wars) 1984;44(5):229-32.

[43] Boytsova YA, Danko SG. EEG differences between resting states with eyes open and closed in darkness. Hum Physiol 2010;36(3):367-9.

[44] Ben-Simon E, Podlipsky I, Okon-Singer H, Gruberger M, Cvetkovic D, Intrator $\mathrm{N}$, et al. The dark side of the alpha rhythm: fMRI evidence for induced alpha modulation during complete darkness. Eur J Neurosci 2013;37(5): 795-803.

[45] Henning S, Merboldt K-D, Frahm J. Task- and EEG-correlated analyses of BOLD MRI responses to eyes opening and closing. Brain Res 2006;1073-1074: 359-64.

[46] Compston A. The Berger rhythm: potential changes from the occipital lobes in man. Brain 2010;133(Pt 1):3-6.

[47] Amochaev A, Salamy A, Alvarez W, Peeke H. Topographic mapping and habituation of event related eeg alpha band desynchronization. Int J Neurosci 1989;49(3-4):151-5.

[48] Dugué L, Marque P, VanRullen R. The phase of ongoing oscillations mediates the causal relation between brain excitation and visual perception. J Neurosci 2011;31(33):11889-93.

[49] Pfurtscheller G, Stancák A, Neuper C. Event-related synchronization (ERS) in the alpha band - an electrophysiological correlate of cortical idling: a review. Int J Psychophysiol 1996;24(1-2):39-46.

[50] Jensen O, Mazaheri A. Shaping functional architecture by oscillatory alpha activity: gating by inhibition. Front Hum Neurosci Front 2010:4:186.

[51] Boroojerdi B, Bushara KO, Corwell B, Immisch I, Battaglia F, Muellbacher W, et al. Enhanced excitability of the human visual cortex induced by short-term light deprivation. Cereb Cortex 2000;10(5):529-34.

[52] Fierro B, Brighina F, Vitello G, Piazza A, Scalia S, Giglia G, et al. Modulatory effects of low- and high-frequency repetitive transcranial magnetic stimulation on visual cortex of healthy subjects undergoing light deprivation. J Physiol 2005;565(2):659-65.

[53] Pitskel NB, Merabet LB, Ramos-Estebanez C, Kauffman T, Pascual-Leone A. Time-dependent changes in cortical excitability after prolonged visual deprivation. NeuroReport 2007;18(16):1703-7.

[54] Stewart LM, Walsh V, Rothwell JC. Motor and phosphene thresholds: a transcranial magnetic stimulation correlation study. Neuropsychologia $2001 ; 39(4): 415-9$

[55] Oever ten S, Schroeder CE, Poeppel D, van Atteveldt N, Zion-Golumbic E. Rhythmicity and cross-modal temporal cues facilitate detection. Neuropsychologia 2014;63:43-50.

[56] Lovelace CT, Stein BE, Wallace MT. An irrelevant light enhances auditory detection in humans: a psychophysical analysis of multisensory integration in stimulus detection. Brain Res Cogn Brain Res 2003;17(2):447-53.

[57] Weisz N, Wühle A, Monittola G. Prestimulus oscillatory power and connectivity patterns predispose conscious somatosensory perception. Proc Nat Acad Sci U. S. A 2014;111(4):E417-25.

[58] Leske S, Ruhnau P, Frey J, Lithari C, Müller N. Prestimulus network integration of auditory cortex predisposes near-threshold perception independently of local excitability. Cereb Cortex 2015;25(12):4898-907.

[59] Machielsen WC, Rombouts SA, Barkhof F, Scheltens P, Witter MP. FMRI of visual encoding: reproducibility of activation. Hum Brain Mapp 2000;9(3): 156-64.

[60] Kosslyn SM, Ganis G, Thompson WL. Neural foundations of imagery. Nat Rev Neurosci 2001;2(9):635-42.

[61] Kosslyn SM, Pascual-Leone A, Felician O, Camposano S, Keenan JP Thompson WL, et al. The role of area 17 in visual imagery: convergent evidence from PET and rTMS. Science 1999;284(5411):167-70.

[62] Sparing R, Mottaghy FM, Ganis G, Thompson WL, Töpper R, Kosslyn SM, et al. Visual cortex excitability increases during visual mental imagery-a TMS study in healthy human subjects. Brain Res 2002;938(1-2):92-7.

[63] Cattaneo Z, Pisoni A, Papagno C, Silvanto J. Modulation of visual cortical excitability by working memory: effect of luminance contrast of mental imagery. Front Psychol Front 2011;2:29. 